\title{
Counseling Framework for HIV-Serodiscordant Couples on the Integrated Use of Antiretroviral Therapy and Pre-exposure Prophylaxis for HIV Prevention
}

\section{Citation}

Morton, J. F., C. Celum, J. Njoroge, A. Nakyanzi, I. Wakhungu, E. Tindimwebwa, S. Ongachi, et al. 2016. "Counseling Framework for HIV-Serodiscordant Couples on the Integrated Use of Antiretroviral Therapy and Pre-exposure Prophylaxis for HIV Prevention." Journal of Acquired Immune Deficiency Syndromes (1999) 74 (Suppl 1): S15-S22. doi:10.1097/QAI.0000000000001210. http://dx.doi.org/10.1097/QAl.0000000000001210.

\section{Published Version}

doi:10.1097/QAI.0000000000001210

\section{Permanent link}

http://nrs.harvard.edu/urn-3:HUL.InstRepos:29739105

\section{Terms of Use}

This article was downloaded from Harvard University's DASH repository, and is made available under the terms and conditions applicable to Other Posted Material, as set forth at http:// nrs.harvard.edu/urn-3:HUL.InstRepos:dash.current.terms-of-use\#LAA

\section{Share Your Story}

The Harvard community has made this article openly available.

Please share how this access benefits you. Submit a story.

\section{Accessibility}




\title{
Counseling Framework for HIV-Serodiscordant Couples on the Integrated Use of Antiretroviral Therapy and Pre-exposure Prophylaxis for HIV Prevention
}

\author{
Jennifer F. Morton, MSW, MPH, * Connie Celum, MD, MPH, *† John Njoroge, MSc, $\S$ \\ Agnes Nakyanzi, BS, || Imeldah Wakhungu, CO,ף Edna Tindimwebwa, DMSCH,\# Snaidah Ongachi, BS,, \\ Eric Sedah, BS, $\mid$ Emmanuel Okwero, BS, $\|$ Kenneth Ngure, MPH, PhD, ** Josephine Odoyo, MPH, 9 \\ Nulu Bulya, MBChB, || Jessica E. Haberer, MD, MS, †† Jared M. Baeten, MD, PhD,*†f \\ and Renee Heffron, PhD, MPH, * for the Partners Demonstration Project Team
}

\begin{abstract}
Background: For HIV-serodiscordant couples, integrated delivery of antiretroviral therapy (ART) for HIV-positive partners and timelimited pre-exposure prophylaxis (PrEP) for negative partners virtually eliminates HIV transmission. Standardized messaging, sensitive to the barriers and motivators to HIV treatment and prevention, is needed for widespread scale-up of this approach.
\end{abstract}

Methods: Within the Partners Demonstration Project, a prospective interventional project among 1013 serodiscordant couples in Kenya and Uganda, we offered ART to eligible HIV-positive partners and PrEP to HIV-negative partners before ART initiation and through the HIV-positive partner's first 6 months of ART use. We conducted individual and group discussions with counseling staff to elicit the health communication framework and key messages about ART and PrEP that were delivered to couples.

From the Departments of *Global Health; †Epidemiology; \$Medicine, University of Washington, Seattle, WA; §Centres for Clinical Research, Kenya Medical Research Institute, Nairobi, Kenya; ||Infectious Disease Institute, Makerere University, Kampala, Uganda; @Microbiology Research, Kenya Medical Research Institute, Nairobi, Kenya; \#Kabwohe Clinical Research Center, Kabwohe, Uganda; **Department of Public and Community Health, Jomo Kenyatta University of Agriculture and Technology, Nairobi, Kenya; and ††Department of Medicine, Massachusetts General Hospital and Harvard Medical School, Boston, MA.

The Partners Demonstration Project was funded by the National Institute of Mental Health of the US National Institutes of Health (Grant R01 MH095507), the Bill \& Melinda Gates Foundation (Grant OPP1056051), and through the generous support of the American people through the US Agency for International Development (cooperative agreement AIDOAA-A-12-00023). Gilead Sciences donated the PrEP medication but had no role in data collection or analysis. The results and interpretation presented here do not necessarily reflect the views of the study funders.

The authors have no conflicts of interest to disclose.

The members of the Partners Demonstration Project Team are listed in Appendix 1 after the References.

Correspondence to: Renee Heffron, PhD, MPH, Departments of Global Health and Epidemiology, University of Washington, 325 Ninth Avenue, Box 359927, Seattle, WA 98104 (e-mail: rheffron@uw.edu).

Copyright $(2016$ Wolters Kluwer Health, Inc. All rights reserved. This is an open-access article distributed under the terms of the Creative Commons Attribution-Non Commercial-No Derivatives License 4.0 (CCBY-NC$\mathrm{ND}$ ), where it is permissible to download and share the work provided it is properly cited. The work cannot be changed in any way or used commercially without permission from the journal.
Results: Counseling sessions for serodiscordant couples about PrEP and ART included discussions of HIV serodiscordance, PrEP and ART initiation and integrated use, and PrEP discontinuation. ART messages emphasized daily, lifelong use for treatment and prevention, adherence, viral suppression, resistance, side effects, and safety of ART during pregnancy. PrEP messages emphasized daily dosing, time-limited PrEP use until the HIV-positive partner sustained 6 months of high adherence to ART, adherence, safety during conception, side effects, and other risks for HIV.

Conclusions: Counseling messages for HIV-serodiscordant couples are integral to the delivery of time-limited PrEP as a "bridge" to ART-driven viral suppression. Their incorporation into programmatic scale-up will maximize intervention impact on the global epidemic.

Key Words: counseling, pre-exposure prophylaxis, antiretroviral treatment, couples, HIV-1 prevention, Africa

(J Acquir Immune Defic Syndr 2017;74:S15-S22)

\section{BACKGROUND}

HIV-serodiscordant couples, in which one partner is HIV-positive and the other is HIV-negative, are estimated to account for up to half of all new HIV infections in Africa and are a priority population for HIV prevention interventions. ${ }^{1}$ Antiretroviral medications, used as antiretroviral therapy (ART) and pre-exposure prophylaxis (PrEP), are potent HIV prevention tools for HIV-serodiscordant couples. ${ }^{2-4}$ Current World Health Organization guidelines recommend ART for all HIV-positive adults upon HIV diagnosis and PrEP as part of combination HIV prevention for people with substantial HIV risk, including HIV-negative partners of serodiscordant couples. ${ }^{4}$

Although the HIV prevention efficacy of ART and PrEP is high, the prevention benefits are only realized when adherence is high., ${ }^{5,6}$ Counseling focused on risk reduction and adherence to prevention tools is an essential component of a comprehensive HIV prevention package, including accompanying ART and PrEP delivery. ${ }^{7}$ Counseling messages that consider multiple levels of the social-ecological model for health behavior have positively impacted HIV 
testing, successful linkage to HIV care and treatment, and adherence to ART. ${ }^{8}$ Counseling sessions with trained staff provide support for couples who are coping with HIVserodiscordancy, encourage engagement in HIV risk reduction, provide valuable information about HIV and routes of transmission, and offer opportunities to identify and problemsolve barriers to adherence. ${ }^{9,10}$

We recently demonstrated that an integrated delivery package of PrEP and ART for HIV-serodiscordant couples in Kenya and Uganda resulted in a $96 \%$ reduction in HIV transmission, a level that was sustained after 2 years of follow-up. ${ }^{11-12}$ In this approach, PrEP was recommended until the HIV-positive partner initiated ART and was virally suppressed, typically by 6 months after ART initiation. ${ }^{2,13}$ An integral component of PrEP and ART delivery was counseling to educate participants about these medications and explore their personal barriers to successful use of them. Thus, we developed a counseling framework with understandable and scalable messages to be provided in counseling sessions accompanying ART and PrEP delivery. Here, we detail these messages so that they can accompany widespread scale up of integrated PrEP and ART for HIV prevention among HIV-serodiscordant couples.

\section{METHODS}

\section{Study Design and Population}

The Partners Demonstration Project was a prospective, open-label implementation study of integrated ART and PrEP delivery for HIV prevention at 4 research sites in Kenya (Thika and Kisumu) and Uganda (Kampala and Kabwohe). The project began in November 2012 and enrolled 1013 serodiscordant couples. To be eligible for enrollment, both members of couples had to be aged $\geq 18$, sexually active with each other, and intending to remain together for at least 1 year. HIV-negative partners had normal renal function and HIV-positive partners had not yet initiated ART. We used a validated risk scoring tool to identify higher-risk couples for this cohort with an expected annual HIV-1 incidence of $>5 \%$ in the absence of integrated delivery of PrEP and ART. ${ }^{14} \mathrm{~A}$ majority of participants (94\%) were married to each other and $14 \%$ of married men reported having more than one wife. Couples were followed quarterly for 24 months (approximately 11 study visits per participant), were encouraged to attend quarterly study visits together, and received couplesbased HIV prevention counseling.

\section{Study Procedures}

At enrollment, HIV-positive participants were counseled to initiate ART in accordance with national guidelines, which initially required a CD4 count of $<350$ cells per microliter or a clinical indication. In 2014, guidelines were expanded to recommend use for all persons with negative partners, regardless of clinical indications. HIV-positive partners had CD4 and HIV viral load testing every 6 months. At every visit, HIV-negative partners were tested for HIV and offered a 3-month supply of PrEP medication [as

S16 | www.jaids.com co-formulated emtricitabine (FTC)/(TDF)]. PrEP discontinuation was encouraged once the HIV-positive partner had used ART for 6 months. Although viral load was measured every 6 months and used to guide counseling when available, counseling on PrEP continuation was not tied to these results because viral load testing was not widely conducted and would not be scalable in public health clinics. Adherence to PrEP was $>80 \%$ for expected doses taken and $>90 \%$ of HIVpositive partners that initiated ART achieved viral suppression within 6 months. ${ }^{11,15}$ PrEP use could extend beyond the 6 months overlap with ART use if couples reported ongoing risk, including additional sexual partners, plans to attempt pregnancy, or concern about the HIV-positive partner's adherence to ART.

PrEP and ART were delivered in the context of HIVserodiscordant couples counseling which used an adaptable, client-centered approach for each couple. The duration and depth of counseling discussions was responsive to the needs of the couple and each individual but sessions were generally 30-45 minutes in length once every 3 months. Counseling sessions were usually conducted by nurse counselors or trained HIV prevention counselors, however, clinicians and pharmacists also delivered the messages when appropriate. As much as appropriate, couples were encouraged to attend counseling sessions together to reap the benefits of a having neutral party hear concerns and challenges about HIV prevention. Participants also met individually a counselor to complete a behavioral questionnaire. Maintenance of participant confidentiality was a key counseling principle; whenever sensitive information (eg, additional partners, concerns about partner ART adherence, or interpersonal violence) was disclosed during behavioral questionnaires, counselors conducted longer individual sessions, provided appropriate referrals, and used information to guide couple-level recommendations including PrEP continuation. Recommendations from counseling staff to prolong PrEP use were not usually questioned by participants. ${ }^{9}$

\section{Development of Counseling Messages \\ Phase I: Message Development}

Our prior work with HIV-serodiscordant couples elicited a great deal of information about barriers and motivators to HIV treatment and prevention that informed the design of the Partners Demonstration Project, the content of messages used during counseling sessions in the project, and normative guidelines for Couples HIV Counseling and Testing (CHCT). ${ }^{16-25}$ In our previous study, HIV-positive partners often had concerns about early ART initiation and did not always accept clinician recommendations to initiate ART, especially those aged $<25$ years and those with higher CD4 counts $\left(>250\right.$ cells per cubic millimeter). ${ }^{26}$ Stigma and fear that ART initiation indicated HIV disease progression and mortality were commonly cited barriers. ${ }^{22,27}$ For HIVserodiscordant couples, the dyad is an important construct that can be leveraged for HIV prevention with members of the couple providing important support to each other in daily pill taking. In the Partners PrEP Study, HIV-negative members of 
FIGURE 1. Framework for counseling HIV-serodiscordant couples about an integrated PrEP and ART strategy. serodiscordant couples demonstrated high adherence to PrEP that may be understood as a function of the desire to reduce their risk while preserving their relationship and maintaining a sexual partnership. ${ }^{17}$ Many serodiscordant couples also report barriers to condom use that are driven by men's preferences and misconceptions about serodiscordance. ${ }^{18}$ Additionally, many serodiscordant couples reported strong desires to conceive that outweighed their fear of HIV transmission and desire to use condoms. ${ }^{18,21}$ However, the desire to conceive and prevent HIV transmission within the partnership and to their infant also increased their motivation to adhere to HIV prevention strategies. ${ }^{20,21,23,27}$

Informed by our previous work with couples, we conducted a scientific literature review to further inform the development of message content. . $17,20-23,26-30^{\text {We used }}$ a conceptual framework that emphasizes the dyadic, contextual conceptualization of HIV prevention behavior ${ }^{31}$ to ensure the content of the messages addressed both individual and relationship factors that could influence uptake of the integrated strategy. Before the beginning of the Partners Demonstration Project, we developed more than 80 standardized messages to accompany the delivery of integrated PrEP and ART. We trained approximately 30 Couples HIV Counseling and Testing-experienced study counselors to deliver the initial messages at all study visits.

\section{Phase II: Message Refinement}

During the second year of the project, we began an iterative process with study counseling staff delivering the messages and intervention to define the content and progression of counseling sessions through messages related to serodiscordance, ART, PrEP, and their integration and to identify key, refined messages using simple and accurate language. We conducted group and individual discussions with project counseling staff at each site to identify counseling topics and the messages most commonly used and of greatest priority, messages that caused participant confusion, common questions, and analogies used to frame the messages to increase participant comprehension. We compared results from these discussions across staff and sites to measure consistency in messages that needed refinement. We conducted 4 iterations of refinement that included editing to ensure appropriateness for the cultural contexts.

\section{Phase III: Message Validation}

After each iteration of message refinement, all project staff were trained on the updated messages, used them with participants, and provided additional feedback on participant reactions to and comprehension of the messages. Additionally, we consulted preliminary results of qualitative work that was simultaneously being undertaken to understand participant preferences and experiences with PrEP and ART ${ }^{9,32}$ to ensure that counseling staff experiences were consistent with participant experiences. Before finalizing the messages, the entire project team reviewed the package of messages and made final revisions.

\section{RESULTS}

\section{Counseling Session Framework}

Counseling sessions followed a framework to facilitate couples understanding of 4 key topic areas: HIV serodiscordance, ART use by the HIV-positive partner, PrEP use by the HIV-negative partner, and the integration of time-limited PrEP and ART to provide a couple with immediate and sustained HIV prevention (Fig. 1). Counseling sessions most often began with a comprehensive discussion of HIV serodiscordance as it was often a confusing concept for participants. Counseling staff emphasized the accuracy of HIV tests, explained that serodiscordance can remain unrecognized for years and did not necessarily indicate infidelity, and confirmed that one partner could remain HIV-negative despite maintenance of a sexual and loving relationship with an HIV-positive partner. 

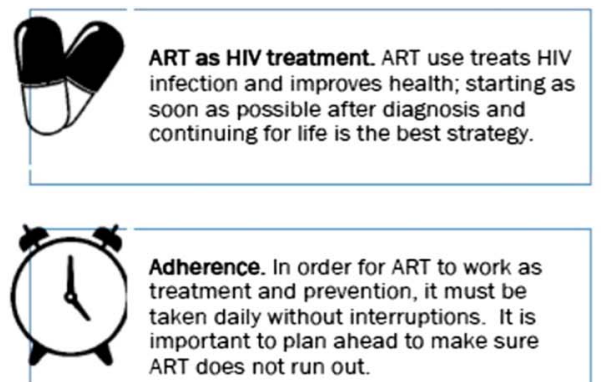

Adherence. In order for ART to work as treatment and prevention, it must be taken daily without interruptions. It is important to plan ahead to make sure ART does not run out.


Side effects. All medications have side effects. Side effects from ART may affect each person differently. Most side effects will lessen after the first 1-2 months of regular use, once the body gets used to the medications.
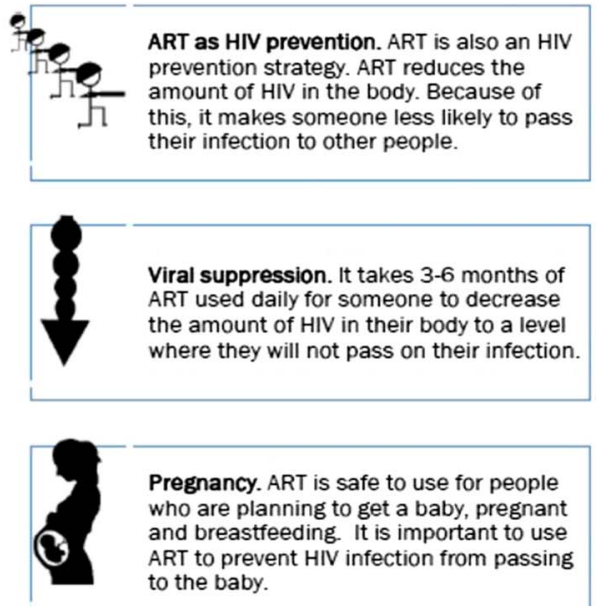

Pregnancy. ART is safe to use for people who are planning to get a baby, pregnant and breastfeeding. It is important to use ART to prevent HIV infection from passing to the baby.

FIGURE 2. ART messages.

\section{ART Messages ART as HIV Treatment}

Messages about the importance of ART use by the HIV-positive partner of the couple were prioritized to both members of the couple as a long-term strategy to treat and prevent HIV (Fig. 2). Staff explained the important clinical benefits of ART for the HIV-positive person, addressed concerns about daily pill taking, and mitigated beliefs that ART initiation should be delayed until symptoms of AIDS developed. ART was framed as a health preserving strategy. HIV-positive partners were encouraged to begin ART as soon as they were willing to reduce the risk for other illnesses and improve overall health.

In addition to recommending immediate ART initiation, daily ART adherence was encouraged without breaks. Counseling staff assisted HIV-positive individuals to foresee challenges with picking up their ART refills and identify ways to overcome these challenges and avoid treatment interruptions. ART adherence was also emphasized to protect against selection of antiviral resistance. Counseling staff explained that interruptions in ART use could result in the development of resistance that would necessitate a change to more complicated second-line ART regimens, which were not always available.

\section{ART as HIV Prevention}

Counseling staff framed the HIV prevention benefits of sustained ART use with high adherence as a commitment to the relationship and the HIV-negative partner. Because understanding treatment as prevention was a challenging concept for most participants, counseling staff developed an analogy to liken ART to an army that was engaged to combat HIV. First, they explained the continuous replication of HIV, measured in blood as "viral load." Then, they described the role of viral load in HIV transmission and how ART reduces viral load, resulting in viral suppression, and thus virtually eliminates risk of HIV transmission. HIV-positive partners were told that it typically takes 3-6 months of daily ART use to decrease the amount of HIV in their blood to a nontransmissible level.

\section{Pregnancy and ART Use}

For couples with pregnancy intentions, counseling staff explained that ART is safe to use during peri-conception, pregnancy, and breastfeeding and that it will not reduce the couple's ability to safely conceive children. HIV-positive women were encouraged to initiate ART before pregnancy to optimize their prepregnancy health, decrease their viral load, and establish a routine with ART use. Messages also emphasized the role of ART to prevent HIV transmission to their babies throughout pre-pregnancy and postpartum.

\section{PrEP Messages}

\section{PrEP Use as HIV Prevention}

Counseling sessions focused on how to use PrEP as an HIV prevention tool for men and women (Fig. 3). Counseling staff emphasized that PrEP is not a life-long intervention, unlike ART, but that it should be used during periods with greatest HIV risk. They encouraged continuous personal risk assessment to identify situations of risk when the option of prolonging PrEP use was most beneficial, including times before the HIV-positive partner had achieved viral suppression, when the couple was attempting pregnancy, and when there were outside risks of HIV transmission from other partners whose HIV status or ART status was unknown.

\section{Appropriate PrEP Use}

Messages stressed the importance of taking PrEP every day to confer protection from HIV and that missed PrEP doses 


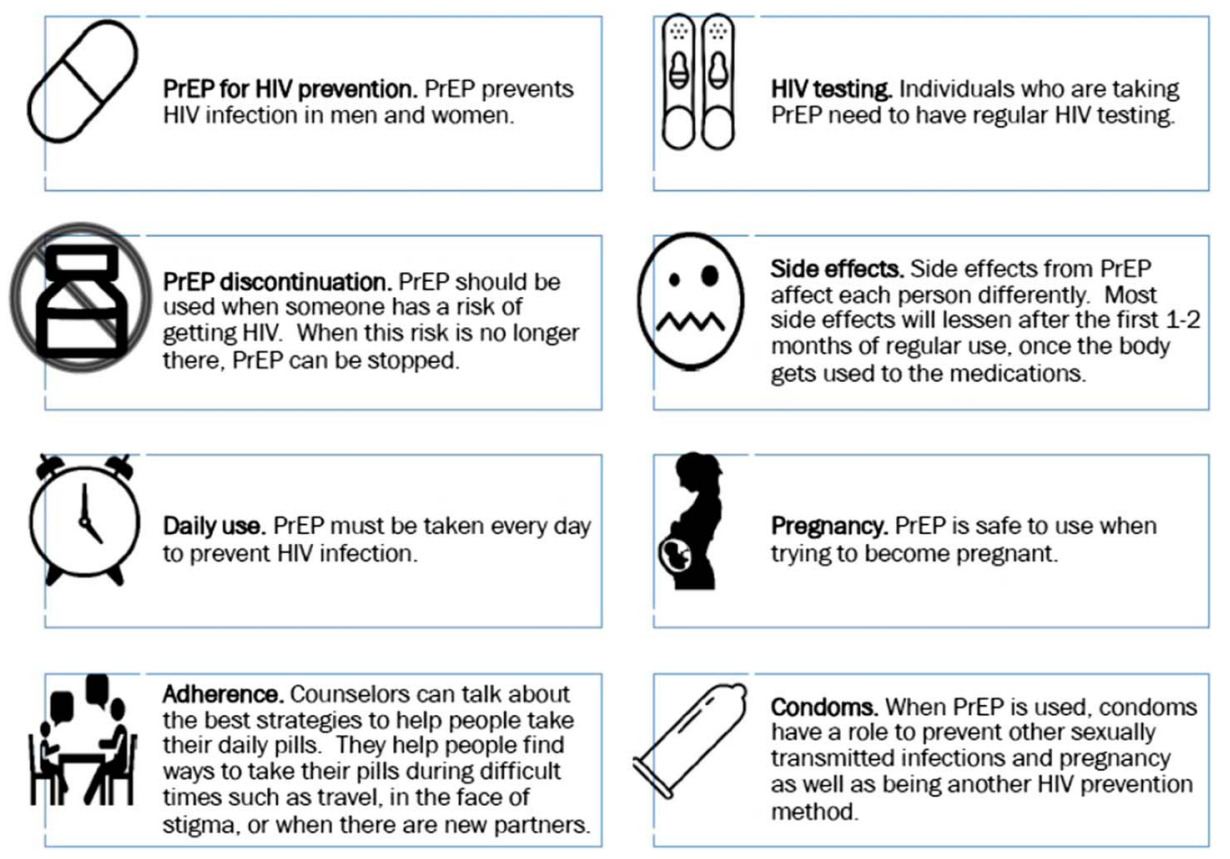

FIGURE 3. PrEP messages.

could render someone vulnerable to HIV infection. Participants were counseled to test for HIV every 3 months to confirm their HIV negative status with additional testing recommended if they suspected that they might be HIV infected. Counseling staff described drug resistance and emphasized that taking PrEP doses while HIV infected could lead to drug resistance, restating the importance of regular testing.

In strategizing with participants about how to attain high medication adherence, counseling staff clarified misconceptions that equated PrEP with a one-time delivered vaccine or beliefs that it can provide protection when only taken after sex. Counseling staff normalized the challenges of high adherence, including stigma of being mistakenly perceived to be HIV positive, travel, or new partners and stressed the importance of identifying strategies to overcome these challenges. Counseling staff also reassured participants that PrEP can be taken without food.

\section{Pregnancy and PrEP Use}

HIV-negative women attempting pregnancy were counseled on the increased risk of HIV transmission during pregnancy and that PrEP is safe to use when they were trying to conceive. Women who became pregnant while using PrEP were encouraged to consider the risks and benefits of continuing PrEP use during pregnancy, including the risk of HIV acquisition if they discontinued PrEP during pregnancy. ${ }^{33}$

\section{Commonalities in PrEP and ART Messages}

To assist participant understanding and prevent confusion, concepts that overlapped in PrEP and ART use were presented using similar language and analogies. For example, counseling staff encouraged all participants to establish a routine time of day for taking their medication, such as a meal time or during a favorite radio show. Participants were encouraged to use cell phone reminders or to ask a friend or their partner to help them remember to take their medication. Counseling staff encouraged participants planning to travel to ensure they had sufficient pills and to keep pills safe in smaller containers for compactness and to avoid drawing attention to the pill bottle.

Participants were frequently concerned they would experience side effects. To minimize fear of side effects, counseling staff explained that all medications have side effects which manifest in each person differently and that side effects to antiretroviral medications are often minimal and lessen after the first 1-2 months of consistent use.

Counseling staff emphasized condom use as an important part of combination prevention and encouraged all participants to use condoms to prevent against sexually transmitted infections and unintended pregnancy. Explanations of the synergistic effects of combination prevention often required additional explanation so counseling staff would provide culturally relevant analogies to help participants understand how condoms work in tandem with PrEP and ART. One such analogy explained that home owners often use multiple forms of security including locks on doors, perimeter walls, and security guards, and that all of these measures work together to protect the home owners from theft and violence. Similarly, one can use ART, condoms, and PrEP to maximize protection against HIV transmission. Because of the overlap in some messages, counselors took care to emphasize and ensure participants' understanding of the unique difference between the medications-specifically, that ART provides lifelong prevention, whereas PrEP is a time-limited prevention intervention insufficient to treat HIV infection. Messages were reinforced when necessary to correct any misinformation and ensure continued understanding. 


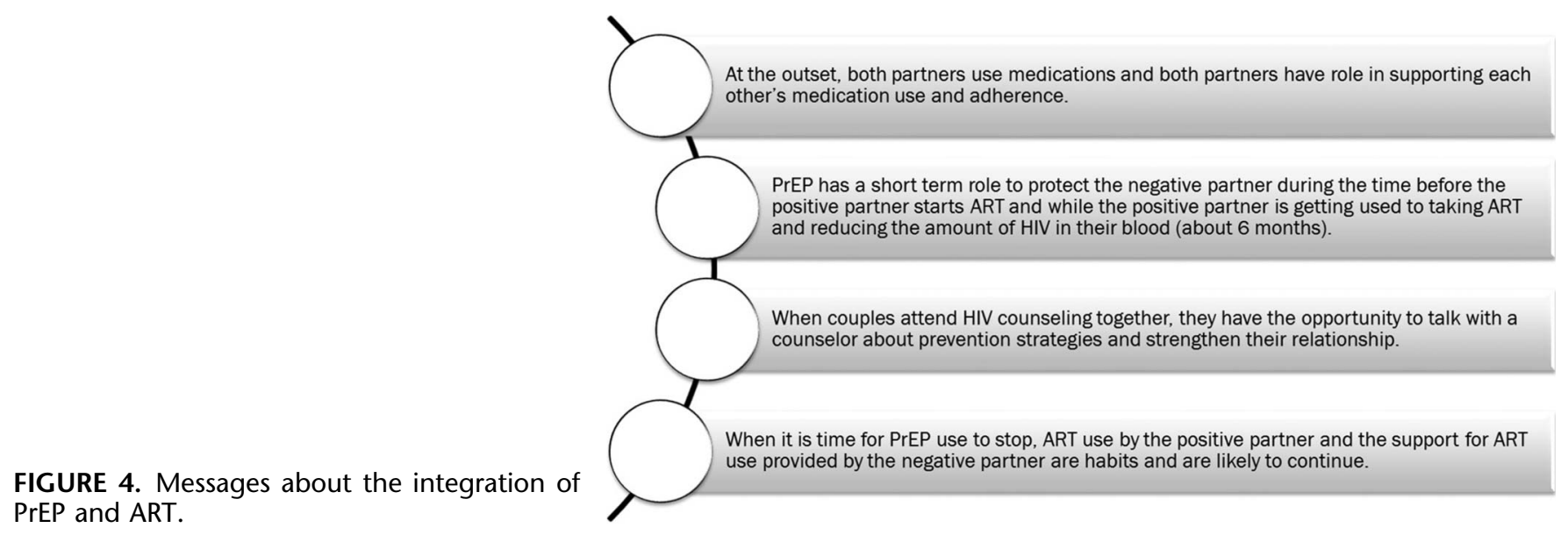

\section{Counseling Messages for the Integrated Delivery of ART and PrEP}

Beginning with first contact with participants and continuing through follow-up, counseling staff explained the integrated use of ART and time-limited PrEP (Fig. 4). Both partners were encouraged to use their respective antiretroviral medications, not to share their medications, and to support each other's adherence. When the HIVpositive partner delayed ART initiation, counseling staff emphasized the importance of high adherence to PrEP to reduce their HIV risk. Once the HIV-positive partner initiated ART, future PrEP discontinuation became a central theme of counseling sessions with counseling staff encouraging PrEP discontinuation after the HIV-positive partner used ART for 6 months, the period of time by which ART would achieve viral suppression. Discussions of PrEP discontinuation often focused on reassuring couples of the effectiveness of ART use by the HIV-positive partner. If either partner expressed concern about viral suppression or the HIV-positive partner's ART adherence, counseling staff could suggest that PrEP be continued for a short period (often extending PrEP for a month at a time). Additionally, counseling staff encouraged PrEP continuation regardless of ART use by the HIV-positive partner if HIV-negative participants disclosed ongoing HIV risk, such as additional partners of unknown HIV/ART status during individual and couples' counseling sessions.

\section{CONCLUSIONS}

To accompany the delivery of integrated PrEP and ART for HIV prevention among HIV-serodiscordant couples, counselors highly experienced in working with HIV-serodiscordant couples identified a framework and the team developed and refined key messages to encourage PrEP use by HIV-negative partners as a "bridge" to sustained ART use by the HIVpositive partner. Each message maps to a common participant concern. ${ }^{9,32}$ The general flow of counseling topics moved from HIV serodiscordance to the purpose of ART use for treatment and prevention, how PrEP works to prevent infection, and how the 2 strategies can work together. Counselors conveyed confidence in the strategies, probed for participant comprehension, and provided responses to outstanding questions. Counseling sessions used a pragmatic approach that minimized burden on the providers and participants and can be delivered by the cadre of lay and professional staff (eg, HIV prevention counselors and nurse counselors) already providing HIV prevention counseling in Kenya and Uganda.

In our work, as well as other studies among HIVserodiscordant couples, the process for couples to understand and accept their HIV-serodiscordant status was complex, requiring time and message repetition during multiple counseling sessions. ${ }^{19}$ When couples learned that they were in a serodiscordant relationship, they were often confused and unsure if their relationship was sustainable. ${ }^{9}$ Client-centered counseling supports the couple by giving them a nonstigmatizing space to understand HIV serodiscordance, explore how their different HIV statuses affect their commitment to each other and the future of their relationship, and identify barriers and facilitators to adopting HIV prevention behaviors. During these sessions, counseling staff can initiate discussions about integrated PrEP and ART as prevention options that often meet couples' short- and long-term needs.

Although the study was not designed to measure the impact of the messages separately from the delivery of integrated PrEP and ART, the framework and accompanying messages were developed to help couples understand how antiretroviral-based HIV prevention works. Participants benefitted from the use of analogies and concise, easy to understand messages to secure their understanding of how ART provides a benefit for both partners and how PrEP provides protection against HIV. Although both partners are taking antiretroviral-based medications, the messages helped counseling staff nurture understanding of how and why PrEP is used as a bridge to effective ART use by the HIV-positive partner, permitting time for both partners to gain confidence in viral suppression as an effective and sufficient HIV prevention strategy and thereby also preparing them for PrEP discontinuation. PrEP as a bridge to ART is an extremely effective HIV prevention strategy ${ }^{11-12}$; the messages were essential to foster understanding and trust that high adherence to the medications prevents HIV transmission. ${ }^{9}$ 
Couples-based counseling incorporating both partners proved beneficial for HIV prevention discussions, including conversations about ART use by the positive partner and PrEP use by the HIV-negative partner. Counseling sessions as a dyad allowed the couple to learn together about HIV, discuss challenges and benefits of ART use with high adherence, and be encouraged to mutually support each other's medication taking. Importantly, these counseling sessions also provide the HIV-negative partner a valuable place to receive support and openly express concerns about HIV risk, including concerns about the positive partner's adherence. Additionally, continued counseling sessions for the couple together and the HIV-negative person alone provide a space for the couple to raise concerns about new sources of risk (eg, interruptions in ART use or new partners) that may indicate re-initiation of PrEP.

Our results are somewhat limited because we did not use a formal development process to create the messages and we did not measure fidelity. However, counseling was an integral component of the highly successful program. Furthermore, messages were developed and refined by a highly experienced counseling staff and responded to concerns participants raised in ancillary qualtitative interviews. ${ }^{9,32}$ The framework and messaging could be strengthened by the use of more formal qualitative research or pilot studies to fully test the utility of these specific messages. In addition, because study participants had very high adherence to both PrEP and ART and couples were only followed for 24 months, messaging about PrEP re-initiation did not emerge as a priority. Future efforts to understand the longevity of use of ART and PrEP in serodiscordant couples would be useful. Finally, many of these messages are relevant for other high risk populations, such as young women, and warrant additional testing among these groups.

The delivery of ART to HIV-positive partners integrated with time-limited PrEP use by HIV-negative partners is a highly effective and cost efficient strategy for HIVserodiscordant couples. ${ }^{34}$ Plans for PrEP delivery to HIVserodiscordant couples are being developed and implemented in place in multiple settings. The counseling framework and accompanying messages used during this demonstration project can be adopted by each program and tailored for the programmatic context to maximize intervention impact on the global epidemic.

\section{ACKNOWLEDGMENTS}

The authors thank the couples who participated in this project for their motivation and dedication and the referral partners, community advisory groups, institutions, and communities that supported this work.

\section{REFERENCES}

1. Dunkle KL, Stephenson R, Karita E, et al. New heterosexually transmitted HIV infections in married or cohabiting couples in urban Zambia and Rwanda: an analysis of survey and clinical data. Lancet. 2008;371:2183-2191.

2. Cohen MS, Chen YQ, McCauley M, et al. Prevention of HIV-1 infection with early antiretroviral therapy. $N$ Engl J Med. 2011;365:493-505.
3. Baeten JM, Donnell D, Ndase P, et al. Antiretroviral prophylaxis for HIV prevention in heterosexual men and women. $N$ Engl J Med. 2012;367: 399-410.

4. World Health Organization. Guideline on When to Start Antiretroviral Therapy and on Pre-Exposure Prophylaxis for HIV. Geneva, Switzerland: World Health Organization; 2015.

5. Grant RM, Anderson PL, McMahan V, et al. Uptake of pre-exposure prophylaxis, sexual practices, and HIV incidence in men and transgender women who have sex with men: a cohort study. Lancet Infect Dis. 2014; 14:820-829.

6. Cottrell ML, Yang KH, Prince HM, et al. A translational pharmacology approach to predicting outcomes of preexposure prophylaxis against HIV in men and women using tenofovir disoproxil fumarate with or without emtricitabine. J Infect Dis. 2016;214:55-64.

7. Coates TJ, Richter L, Caceres C. Behavioural strategies to reduce HIV transmission: how to make them work better. Lancet. 2008;372:669-684.

8. Babalola S, Van Lith LM, Mallalieu EC, et al. A framework for health communication across the HIV treatment continuum. J Acquir Immune Defic Syndr. 2017;74(suppl 1):S5-S14.

9. Ngure, Heffron R, Curran K, et al. I knew I would be safer. experiences of Kenyan HIV-serodiscordant couples soon after pre-exposure prophylaxis (PrEP) initiation. AIDS Patient Care STDS. 2016;30:78-83.

10. Coates TJ. An expanded behavioral paradigm for prevention and treatment of HIV-1 infection. J Acquir Immune Defic Syndr. 2013;63: S179-S182.

11. Baeten JM, Heffron R, Kidoguchi L, et al. Integrated delivery of antiretroviral treatment and pre-exposure prophylaxis to HIV-1serodiscordant couples: a prospective implementation study in Kenya and Uganda. PLoS Med. 2016;13:e1002099.

12. Baeten J, Heffron R, Kidoguchi L, et al. Integrated delivery of PrEP and ART results in sustained near elimination of HIV transmission in African HIV-serodiscordant couples: final results from the Partners Demonstration Project. Paper presented at: 21st International AIDS Conference (AIDS 2016) 18-22 July 2016. Durban, South Africa.

13. Mujugira A, Celum C, Coombs RW, et al. HIV transmission risk persists during the first 6 months of antiretroviral therapy. J Acquir Immune Defic Syndr. 2016;72:579-584.

14. Kahle EM, Hughes JP, Lingappa JR, et al. An empiric risk scoring tool for identifying high-risk heterosexual HIV-1-serodiscordant couples for targeted HIV-1 prevention. J Acquir Immune Defic Syndr. 2013;62:339-347.

15. Mujugira A, Baeten J, Kidoguchi L, et al. Sustained viral suppression among East African HIV-infected members of serodiscordant couples initiating ART with high CD4 counts and during pregnancy. Paper presented at: 11th International Conference on HIV Treatment and Prevention Adherence, 9-11 May 2016, 2016. Fort Lauderdale, Florida.

16. Ndase P, Celum C, Thomas K, et al. Outside sexual partnerships and risk of HIV acquisition for HIV uninfected partners in African HIV-serodiscordant partnerships. J Acquir Immune Defic Syndr. 2012;59:65-71.

17. Ware NC, Wyatt MA, Haberer JE, et al. What's love got to do with it? Explaining adherence to oral antiretroviral pre-exposure prophylaxis for HIV-serodiscordant couples. J Acquir Immune Defic Syndr. 2012;59:463-468.

18. Ngure K, Mugo N, Celum C, et al. A qualitative study of barriers to consistent condom use among HIV-1 serodiscordant couples in Kenya. AIDS care. 2012;24:509-516.

19. Curran K, Baeten JM, Coates TJ, et al. HIV-1 prevention for HIV-1 serodiscordant couples. Curr Hiv-Aids Rep. 2012;9:160-170.

20. Mujugira A, Heffron R, Celum C, et al. Fertility intentions and interest in early antiretroviral therapy among East African HIV-1-infected individuals in serodiscordant partnerships. J Acquir Immune Defic Syndr. 2013; 63:e33-35.

21. Pintye J, Ngure K, Curran K, et al. Fertility decision-making among Kenyan HIV-serodiscordant couples who recently conceived: implications for safer conception planning. Aids Patient Care St. 2015;29: 510-516.

22. Curran K, Ngure K, Shell-Duncan B, et al. "If I am given antiretrovirals I will think I am nearing the grave": Kenyan HIV-serodiscordant couples' attitudes regarding early initiation of antiretroviral therapy. AIDS. 2014; 28:227-233.

23. Ngure K, Baeten JM, Mugo N, et al. My intention was a child but I was very afraid: fertility intentions and HIV risk perceptions among HIVserodiscordant couples experiencing pregnancy in Kenya. AIDS Care. 2014;26:1283-1287. 
24. Ware NC, Pisarski EE, Haberer JE, et al. Lay social resources for support of adherence to antiretroviral prophylaxis for HIV prevention among serodiscordant couples in Sub-Saharan Africa: a qualitative study. AIDS Behav. 2015;19:811-820.

25. Centers for Disease Control and Prevention Center for Global Health, The Task Force for Global Health. Couples HIV Testing and Counseling (CHCT) in Health Care Facilities Atlanta, GA: Centers for Disease Control and Prevention (CDC); 2011.

26. Mujugira A, Celum C, Thomas KK, et al. Delay of antiretroviral therapy initiation is common in East African HIV-infected individuals in serodiscordant partnerships. J Acquir Immune Defic Syndr. 2014;66: 436-442.

27. Heffron R, Ngure K, Mugo N, et al. Willingness of Kenyan HIV-1 serodiscordant couples to use antiretroviral-based HIV-1 prevention strategies. J Acquir Immune Defic Syndr. 2012;61:116-119.

28. Celum C, Wald A, Lingappa JR, et al. Acyclovir and transmission of HIV-1 from persons infected with HIV-1 and HSV-2. $N$ Engl J Med. 2010;362:427-439.

29. Lingappa JR, Petrovski S, Kahle E, et al. Genomewide association study for determinants of HIV-1 acquisition and viral set point in HIV-1 serodiscordant couples with quantified virus exposure. PLoS One. 2011; 6:e28632.

30. Carroll JJ, Ngure K, Heffron R, et al. Gendered differences in the perceived risks and benefits of oral PrEP among HIV-serodiscordant couples in Kenya. AIDS Care. 2016:1-7.

31. Karney BR, Hops H, Redding CA, et al. A framework for incorporating dyads in models of HIV-prevention. AIDS Behav. 2010;14(suppl 2): 189-203.

32. Wyatt MA, Pisarski EE, Tam M, et al. Experiences of PrEP discontinuation in African HIV-serodiscordant couples: qualitative results from the partners demonstration project. Paper presented at: 21st International AIDS Society (AIDS 2016) 18-22 July 2016. Durban, South Africa.
33. Centers for Disease Control and Prevention. Interim guidance for clinicians considering the use of preexposure prophylaxis for the prevention of HIV infection in heterosexually active adults. MMWR Morb Mortal Wkly Rep. 2012;61:586-589.

34. Ying R, Sharma M, Heffron R, et al. Cost-effectiveness of pre-exposure prophylaxis targeted to high-risk serodiscordant couples as a bridge to sustained ART use in Kampala, Uganda. J Int AIDS Soc. 2015;18(4 suppl 3):20013.

\section{APPENDIX 1. Partners Demonstration Project Team}

Coordinating Center (University of Washington) and collaborating investigators (Harvard Medical School, Johns Hopkins University, Massachusetts General Hospital): Jared Baeten (protocol chair), Connie Celum (protocol co-chair), Renee Heffron (project director), Deborah Donnell (statistician), Ruanne Barnabas, Jessica Haberer, Harald Haugen, Craig Hendrix, Lara Kidoguchi, Mark Marzinke, Susan Morrison, Jennifer Morton, Norma Ware, Monique Wyatt.

\section{Project sites:}

Kabwohe, Uganda (Kabwohe Clinical Research Centre): Stephen Asiimwe, Edna Tindimwebwa.

Kampala, Uganda (Makerere University): Elly Katabira, Nulu Bulya.

Kisumu, Kenya (Kenya Medical Research Institute): Elizabeth Bukusi, Josephine Odoyo.

Thika, Kenya (Kenya Medical Research Institute, University of Washington): Nelly Rwamba Mugo, Kenneth Ngure.

Data Management was provided by DF/Net Research, Inc. (Seattle, WA). PrEP medication was donated by Gilead Sciences. 